\title{
Prevention of hepatitis B virus-related hepatocellular carcinoma
}

\author{
Chih-Lin Lin ${ }^{1,2}$, Jia-Horng Kao ${ }^{3,4,5}$ \\ 'Department of Gastroenterology, Renai branch, Taipei City Hospital, Taipei 10629, Taiwan. \\ 2Department of Psychology, National Chengchi University, Taipei 116011, Taiwan. \\ ${ }^{3}$ Graduate Institute of Clinical Medicine, National Taiwan University, College of Medicine, Taipei 10002, Taiwan. \\ ${ }^{4}$ Department of Internal Medicine, National Taiwan University Hospital, Taipei 10002, Taiwan. \\ ${ }^{5}$ Hepatitis Research Center, and Department of Medical Research, National Taiwan University, National Taiwan University \\ Hospital, Taipei 10002, Taiwan.
}

Correspondence to: Prof. Jia-Horng Kao, Graduate Institute of Clinical Medicine, National Taiwan University, College of Medicine, 1Chang-Te St., Taipei 10002, Taiwan. E-mail: kaojh@ ntu.edu.tw

How to cite this article: Lin CL, Kao JH. Prevention of hepatitis B virus-related hepatocellular carcinoma. Hepatoma Res $2021 ; 7: 9$. http://dx.doi.org/10.20517/2394-5079.2020.125

\author{
Received: 15 Oct 2020 First Decision: 16 Nov 2020 Revised: 24 Nov 2020 Accepted: 30 Nov 2020 Published: 7 Jan 2021 \\ Academic Editor: Antonio Bertoletti Copy Editor: Cai-Hong Wang Production Editor: Jing Yu
}

\begin{abstract}
Hepatocellular carcinoma (HCC), especially hepatitis B virus (HBV)-related, remains a major cause of cancerrelated mortality worldwide. Unless there is early detection with curative treatment, the 5-year survival rate of advanced HCC is less than $15 \%$. The preventive strategies for HBV-related HCC are thus urgently needed to reduce the global burden of this disastrous cancer. Primary prevention involves the avoidance of viral infection through hepatitis $B$ vaccination and interruption of viral transmission from patients with chronic HBV infection. Universal neonatal hepatitis B vaccination program has successfully reduced the prevalence of HBV carriage rate as well as HCC incidence in vaccinated cohorts. However, HBV elimination is still difficult to achieve. Regarding secondary prevention, long-term treatment with nucleos(t)ide analogues has been proven to reduce the risk of HBV-related HCC. Individual risk stratification and a periodic HCC surveillance in these patients could facilitate early HCC diagnosis. Finally, tertiary prevention can also be achieved by life-long treatment with NAs to reduce the risk of HCC recurrence after curative treatment of primary HCC. Challenges ahead include the fact that HBV is not yet curable by current antiviral agents. Combination therapy with direct anti-HBV agents and host-targeting immunomodulatory agents is under active development. In addition, HCC risk cannot be eliminated even in patients with $\mathrm{HBsAg}$ seroclearance or functional cure. Therefore, $\mathrm{HCC}$ surveillance is strongly recommended for every patient with chronic HBV infection.
\end{abstract}

Keywords: Anti-HBV therapy, hepatitis B vaccination, hepatitis B virus, hepatocellular carcinoma

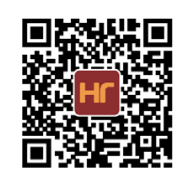




\section{INTRODUCTION}

Hepatocellular carcinoma (HCC) is one of the most prevalent solid tumors and leading causes of cancerrelated death worldwide. In 2018, the estimated annual incidence and mortality increased to 841,000 cases and 782,000 deaths, respectively ${ }^{[1]}$. The highest HCC incidence rates are found in East and Southeast Asia and Northern Africa as well, but the incidence rates of HCC also increased in North America and Europe over the past few decades ${ }^{[2]}$. Although early detection by HCC surveillance provides the opportunity of curative treatment for early-stage HCC, the worldwide 5-year survival rate of HCC has been unsatisfactory over the past two decades ${ }^{[3]}$. In the last 10 years, emerging molecular target therapy and immunotherapy has constituted a major breakthrough for advanced HCC. However, overall survival has improved marginally ${ }^{[4]}$. The major reasons for the poor prognosis of HCC include the heterogeneous nature of HCC affecting treatment outcome and the majority of HCC occurring in countries where medical resources $\operatorname{are~limited~}^{[5]}$. Therefore, we urgently need effective measures to prevent the occurrence of HCC in the populations at risk. The current exploration of HCC risk factors provides us a good opportunity to develop preventive strategies. It is known that the etiologies of HCC include virological factors (such as hepatitis virus infection), chemical factors (such as aflatoxin B1 and alcohol) and genetic as well as metabolic factors [such as hereditary hemochromatosis, metabolic associated fatty liver disease (MAFLD) or non-alcoholic steatohepatitis (NASH) $]^{[6,7]}$. The contribution of different risk factors to HCC varies widely by country and region. Among them, chronic hepatitis B virus (HBV) infection is one of the most important risk factors. The global burden of HCC in 2015 revealed that the contribution of HBV to HCC mortality was geographically related. From highest (up to 40\%-60\%) in most areas of Asia and Sub-Saharan Africa to the less common cause of HCC death, approximately $9 \%$ in North America ${ }^{[8]}$.

Since 1981, the development of hepatitis B vaccines has successfully prevented the spread of HBV across the world. In addition, the development of anti-HBV agents has led to a delay or block of the progression of chronic hepatitis B (CHB). As a consequence, prevention of HBV-related HCC in this setting should be expected by early 2050s. In this review, the mechanisms of HBV-induced HCC and individual prevention of HBV-related HCC will be summarized and discussed.

\section{FACTORS ASSOCIATED WITH HBV-RELATED HCC}

Thanks to the advances in the molecular biology of HBV, the pathogenesis of HBV-related HCC is largely clarified [Figure 1]. The pathogenesis of HCC in patients with CHB can be divided according to HBVrelated direct and indirect mechanisms. Direct oncogenic effects of HBV include HBV-host genome integration and HBV-encoded oncogenic protein. The integration of HBV DNA into the host genome induces both host chromosomal instability and insertional mutagenesis of HCC-related genes ${ }^{[9]}$. This integration in the host genome most frequently happens in the telomerase reverse transcriptase region and myeloid/lymphoid or mixed-lineage leukemia 4 gene. Dysregulation of telomerase and histone methyltransferase expression subsequently increase the risk of hepatocarcinogenesis ${ }^{[10]}$. Truncated HBV pre-S/S mutation induces surface protein synthesis imbalance and may increase endoplasmic reticulum stress and decrease tumor suppressor gene expression, and thus, it may play a primary role in oncogenesis ${ }^{[11]}$. Among HBV-encoded proteins, HBV X protein has the most oncogenic effect in hepatocarcinogenesis. HBV X protein is a multifunctional regulator, and it is involved in several cancerrelated molecular mechanisms, including alteration of signal pathways (e.g., inhibition of P53 and activation of the Jak1/STAT pathway), DNA repair (e.g., alteration of p53/ERCC3), apoptosis (inhibition of P53), mitochondrial function (e.g., activation of calcium-dependent kinase pathway, epigenetic modification (e.g., epigenetic silencing of ASPP gene), and expression of noncoding RNA (e.g., activation of the NF- $\mathrm{B}$ pathway $)^{[12,13]}$. Through these mechanisms, HBV X protein directly contributes to HCC development.

Hepatitis B viral load is the strongest risk factor associated with an increased HCC risk, independent of HBeAg status and cirrhosis. Chronic inflammation caused by long-term exposure to high levels of HBV 


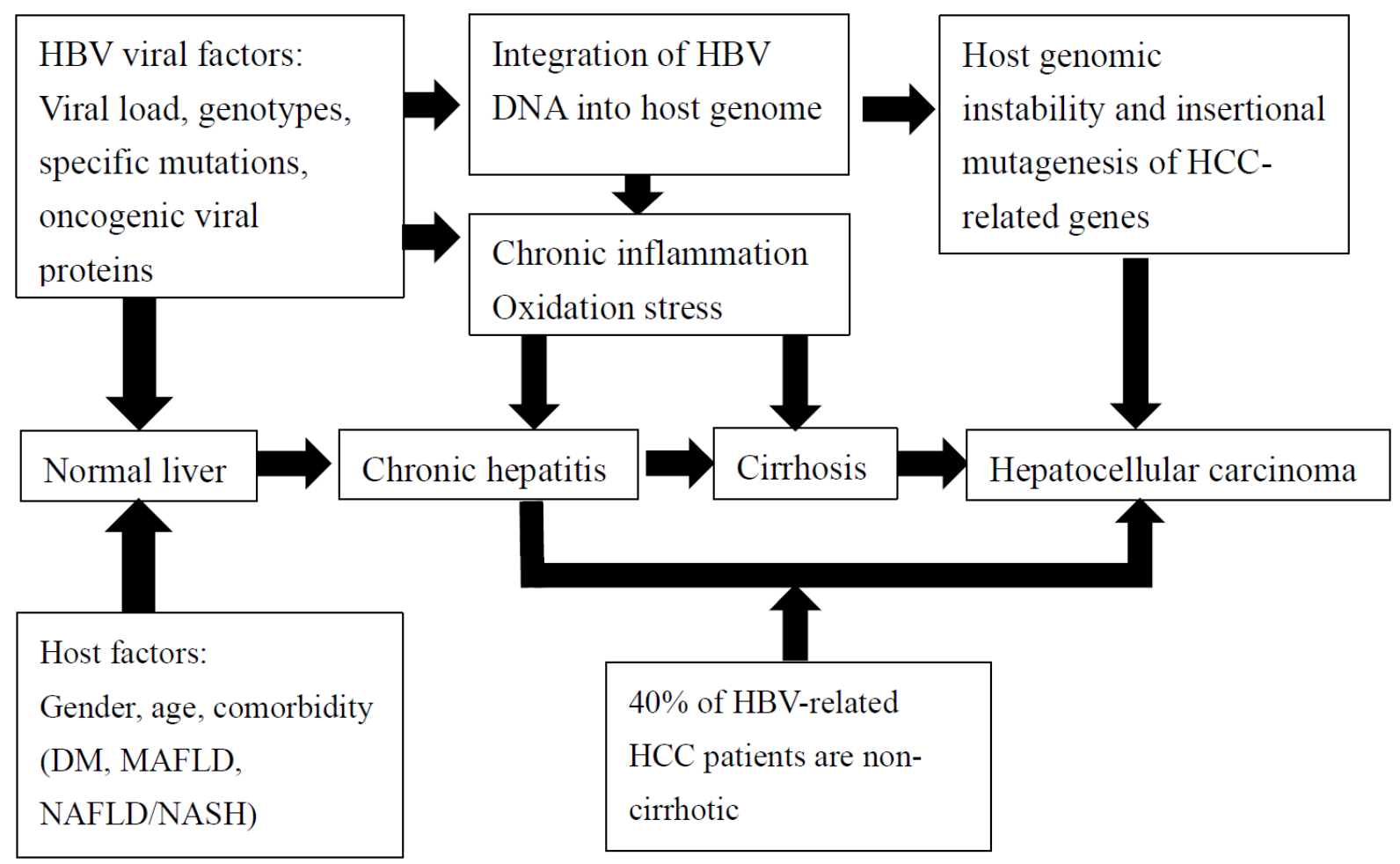

Figure 1. Factors associated with hepatitis $B$ virus-related hepatocarcinogenesis. DM: diabetes mellitus; HBV: hepatitis B virus; HCC: hepatocellular carcinoma; MAFLD: metabolic associated fatty liver disease; NAFLD: non-alcoholic fatty liver disease; NASH: nonalcoholic steatohepatitis

DNA will increase the risk of subsequent HCC. Clinically, several cross-sectional and longitudinal cohort studies in Asia revealed a positive correlation between serum HBV DNA level and HCC development ${ }^{[1-20]}$. One of the most important longitudinal studies tracking HBV DNA and risk of HCC is the REVEAL-HBV study. REVEAL-HBV study providing convincing evidence of a dose-response relationship between HBV DNA levels and risk of HCC development in HBeAg-negative carriers. In the natural course, patients with persistently high HBV viremia had the greatest risk of HCC development ${ }^{[14]}$.

After a long-time evolution, at least $10 \mathrm{HBV}$ genotypes (A to J) with distinct geographic and ethnic distributions have been identified ${ }^{[21]}$. Briefly, genotypes B and C are highly prevalent in the Asia-Pacific region. Genotypes A and D are widespread in Africa, Europe and North America. The risk of HCC is significantly different between HBV genotypes. Several studies from Asian countries have revealed that patients with genotype $\mathrm{C}$ infection have a greater risk of developing HCC than those with genotype B infection ${ }^{[20-23]}$. In a prospective study, Yu et al. ${ }^{[20]}$ found that genotype $\mathrm{C}$ patients had significantly higher serum levels of HBV DNA than did genotype B patients. Furthermore, genotype C with high viral load had a substantial increase in HCC risk than those with genotype B with low viral load. Thus, through chronic inflammation caused by high HBV viral load and its interaction with host immunity, an increased risk of $\mathrm{HCC}$ can be predicted in HBV genotype C patients. In the regions where genotypes A and D are prevalent, the comparison of HCC risk between patients with genotype A and D infection remains controversial. A meta-analysis including more than 14,500 patients revealed that the risk of HCC was similar between genotypes $\mathrm{A}$ and $\mathrm{D}^{[24]}$. On the contrary, in a cohort study of Alaska native persons with HBV genotype A, $\mathrm{B}, \mathrm{C}, \mathrm{D}$ and $\mathrm{F}$ revealed that individuals with genotype $\mathrm{A}, \mathrm{C}$ and $\mathrm{F}$ had significantly higher HCC risk than those with genotype $\mathrm{B}$ and $\mathrm{D}^{[25]}$. These studies supported the notion that HBV genotypes show a strong correlation with HCC risk. Beside HBV genotype, mutations in specific regions are also related to HCC development. Core promoter mutations, especially basal core promoter mutations, are confirmed to be 
associated with an increased risk of $\mathrm{HCC}^{[21,23,26]}$. Pre-S deletion mutations may block hepatocyte DNA repair and lead to genomic instability ${ }^{[27]}$. Taken together, these naturally occurring mutations may contribute to hepatocarcinogenesis in patients with chronic HBV infection.

The indirect mechanism of HBV-related hepatocarcinogenesis is through the process of chronic necroinflammation, regeneration and fibrosis, associated with HBV or concomitant HBV and other causes of inflammation, such as MAFLD or nonalcoholic fatty liver disease (NAFLD). Although several studies have reported that the prevalence of NAFLD is lower in HBV carriers than general population ${ }^{[28]}$, the concurrent NAFLD increases disease progression and the risk of $\mathrm{HCC}$ in $\mathrm{CHB}^{[29,30]}$. In particular, NAFLD was also associated with HCC development in CHB patients with effective anti-HBV therapy ${ }^{[31]}$.

Regarding non-viral factors, a systematic review revealed that smoking as an environmental factor augmented HCC development with an odds ratio of 1.90 (95\%CI: $1.68-2.14 ; P<0.00001)^{[32]}$. In a recent study from Taiwan, Wang et al. ${ }^{[33]}$ further found that the plasma levels of interferon-g and natural killer cell levels in HBV carriers who smoked were significantly lower than in those who did not. Through the reduction of antiviral immunity, HBV carriers who smoked had persistently higher viral load and serum ALT levels compared with nonsmokers over the long-term follow-up period.

Finally, host predisposing genetic variations also contribute to HCC risk in HBV carriers. The application of genome-wide association study has identified several susceptibility loci or genetic variants for HBVrelated HCC. Recent uncovered host genetic variants associated with HBV-related HCC included HLA class II region, KIF1B gene, STAT4 gene and GRIK1 gene ${ }^{[34]}$. Genomic studies also elucidated a number of somatic mutated genes affecting signal pathways associated with HBV-related HCC. For example, CTNNB1 mutation in the Wnt/b-catenin pathway was associated with hepatic cell proliferation and differentiation. Mutations of TP53 in the cell cycle pathway was associated with genomic stability ${ }^{[10]}$. However, functional assessment is warranted to confirm the causal relationship with HBV-related HCC.

\section{PREVENTIVE STRATEGIES FOR HBV-RELATED HCC}

In the past few years, the incidence and mortality of HBV-related HCC has continuously increased ${ }^{[1]}$. Thus, efforts in the development of prevention measures is urgently needed to decrease the global burden of HBV-related HCC. From the literature, the three levels of preventive strategies, including primary, secondary, and tertiary prevention will be effective for the prevention of HBV-related HCC [Figure 2].

\section{Primary prevention}

The goals of primary prevention of HBV-related HCC include the avoidance of HBV infection among susceptible populations and the interruption of mother-to-child transmission (MTCT) of HBV.

\section{HBV vaccination}

Enhancement of host immunity by vaccination remains the most effective way for the prevention of HBV infection ${ }^{[35]}$. Since the development of the first hepatitis B vaccine in 1981, universal infant HBV vaccination has been introduced in 187 countries $^{[36]}$ with $75 \%$ of the global coverage rate among infants ${ }^{[37]}$. After hepatitis B vaccine was implemented, the prevalence of chronic HBV infection was markedly reduced in most countries. For example, Taiwan is a hyperendemic area for HBV infection, where the prevalence of HBsAg was found to be more than $12 \%$ in the general population ${ }^{[38]}$. After the implementation of the hepatitis B vaccination program in $1984^{[39]}$, the prevalence of chronic HBV infection rapidly changed from $10.9 \%$ in children who were born before 1984 to $0.5 \%$ in children who were born after $1984^{[40]}$. This dramatic change also appeared in countries where universal infant HBV vaccination was adopted. The global prevalence of HBV in children decreased from $4.7 \%$ before vaccine adoption to $1.3 \%$ in $2015^{[41]}$. Surprisingly, the incidence of HCC in children also decreased after implementation of universal infant 


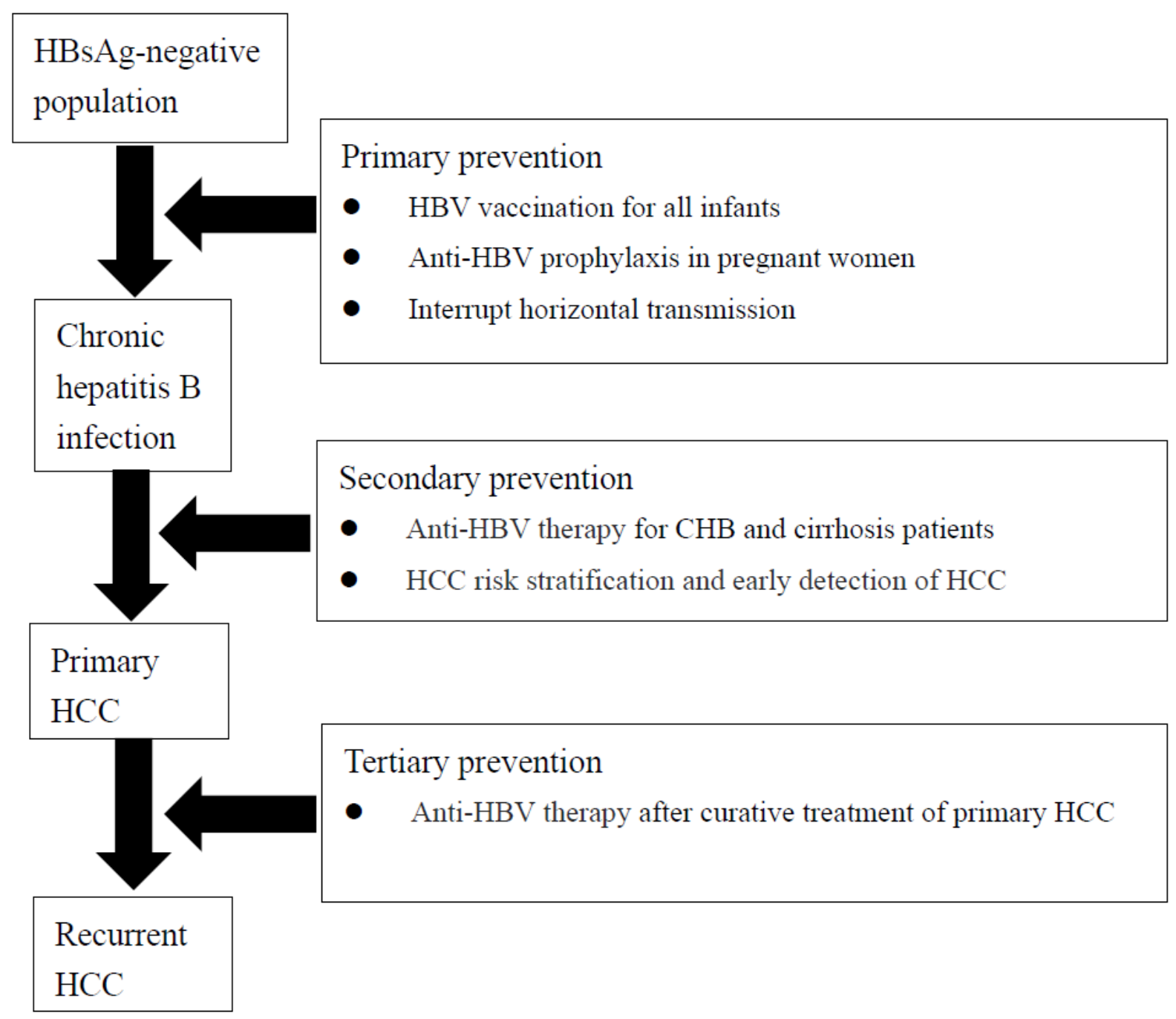

Figure 2. Preventive strategies of HBV-related HCC. HBV: hepatitis B virus; HCC: hepatocellular carcinoma

HBV vaccination. In Taiwan, the incidence of HCC in children was 0.23 per 100,000 person-years in the vaccinated children, compared with 0.92 per 100,000 person-years in the unvaccinated cohorts ${ }^{[42]}$. According to the population-based cancer registry in Taiwan, the annual incidence of HCC increased slightly, 1.3\%, in adults during 2003 to 2011. In contrast, the annual incidence of HCC decreased 16.6\% in children during the same period and decreased to zero in $2011^{[43]}$. Similarly, HCC incidence significantly decreased $84 \%$ in China ${ }^{[44]}, 70 \%$ in Korea ${ }^{[45]}$ and down to zero in Alaska ${ }^{[46]}$ after implementation of a national hepatitis B vaccination program. Ample evidence from Taiwan and other countries revealed that the hepatitis B vaccine significantly reduces the incidence of HCC in children, teenagers and young adults. Therefore, hepatitis B vaccine has been recognized as the first anti-cancer vac the history of medical development ${ }^{[47]}$.

\section{Antiviral prophylaxis in pregnant women}

Despite that effective HBV vaccination dramatically decreased the prevalence of $\mathrm{HBV}$ in children in the post-vaccine era ${ }^{[41]}$, MTCT of HBV has not been completely blocked. In a prospective study which enrolled 303 mother-infant pairs, all of the mothers were HBV carriers, and the infants received a complete immunization program. There were $3.3 \%$ of children with chronic HBV infection. All of the children with chronic HBV infection were born to HBeAg-positive mothers with high viral load. In addition, the risk of 
MTCT of HBV was positively correlated with maternal viral load ${ }^{[48]}$. To completely block MTCT of HBV, anti-HBV therapy for HBsAg-positive mothers was suggested. Afterwards, two prospective studies from Taiwan and China enrolled HBeAg-positive pregnant women with high HBV DNA levels. Anti-HBV prophylaxis with tenofovir disoproxil fumarate (TDF) $300 \mathrm{mg}$ daily was administrated in the third trimester of pregnancy. Compared to the mothers without anti-HBV prophylaxis, the infants born to mothers with TDF prophylaxis had significantly lower rates of HBV infection ${ }^{[49,50]}$. Currently, antiviral prophylaxis in the third trimester of pregnancy in HBsAg-positive mothers with high viral load is recommended in international liver societies ${ }^{[11,52]}$.

\section{Interruption of horizontal transmission}

Although universal HBV vaccination is the key to primary HBV prevention, it is equally important to prevent horizontal HBV transmission in adults. HBV vaccination for individuals at risk of contracting HBV infection, including healthcare workers, people who inject drugs, men who have sex with men, HIV carriers and sex workers, is effective in interrupting horizontal transmission. A new hepatitis B vaccine with simple schedule, only two doses over one month, can increase patient compliance and coverage in adults ${ }^{[53]}$. In addition, appropriate information to promote safer sex and intention of receiving HBV vaccination also aids in the effort to prevent horizontal HBV transmission.

\section{Secondary prevention}

Secondary prevention for HBV-related HCC involves anti-viral treatment to delay or prevent liver disease progression and early development of HCC.

\section{Anti-HBV therapy}

Currently, standard or pegylated interferon- $\alpha$ (PEG-IFN- $\alpha$ ) and NAs are widely approved for the anti-HBV treatment of $\mathrm{CHB}^{[51,52,54]}$. According to inhibitory efficacy and resistance incidence, the first-line treatments include PEG-IFN- $\alpha$, entecavir (ETV), tenofovir disoproxil fumarate (TDF) and tenofovir alafenamide ${ }^{[55]}$. Several earlier randomized control studies and meta-analyses reported that interferon- $\alpha$ and PEG-IFN- $\alpha$ therapy reduced the risk of CHB progression and HCC incidence by $30 \%-40 \%{ }^{[56-59]}$. Regarding NAs, the prevention effect in HBV-related HCC in cirrhosis patients is more obvious. The cohort studies from Taiwan, Japan and Hong Kong demonstrated that CHB patients with ETV therapy had significantly lower cumulative HCC incidence, with HCC risk reduction by $45 \%-60 \%{ }^{[60]}$, especially in a cirrhosis setting. A recent study enrolled 1,088 patients with and without TDF treatment in Hong Kong. Compared to untreated patients, TDF treatment significantly reduced risks of HCC [adjusted hazard ratio (aHR) 0.46, $P$ $<0.01]^{[61]}$. Similarly, Nguyen et al ${ }^{[62]}$ conducted a retrospective study in which $6,914 \mathrm{CHB}$ patients with and without TDF treatment were recruited. During 8 years of follow-up, TDF treatment had a $77 \%$ reduction in the risk of HCC (aHR, 0.23; 95\%CI: 0.56-0.92) in cirrhotic patients and a 73\% reduction $(\mathrm{aHR}=0.27$; 95\%CI: 0.07-0.98) in non-cirrhotic patients. According to the national healthcare database in Taiwan, Hsu et al. ${ }^{[63]}$ found that the HCC annual incidence decreased with an adjusted incidence rate ratio of 0.73 (95\%CI: 0.66-0.80) per year in patients receiving ETV or TDF treatment.

Recently, several studies have compared the preventive effects for HBV-related HCC of first-line NAs. A Korean nationwide cohort study revealed that patients with TDF treatment had a significantly lower risk of HCC $(\mathrm{HR}=0.61 ; 95 \% \mathrm{CI}: 0.54-0.70)$ than patients with ETV treatment ${ }^{[64]}$. In a meta-analysis that included seven articles with 3,698 patients, the incidence of HCC was significantly lower among patients with TDF treatment than those with ETV treatment (rate ratio: 0.66; 95\%CI: 0.49-0.89; $P=0.008)^{[65]}$. However, three hospital-based studies in Taiwan and Korea demonstrated that the annual HCC incidence was not different between the patients receiving ETV and TDF treatment ${ }^{[6-68]}$. The recent international study including 19 centers from 6 countries or regions also revealed that TDF and ETV had a similar effect in the prevention of $\mathrm{HCC}$ in patients with $\mathrm{CHB}^{[69]}$. In the latest meta-analysis investigating 31 studies that enrolled 119,053 
patients, the incidence of HCC was similar in patients with TDF or ETV treatment ${ }^{[70]}$. Taking these lines of evidence together and unless we could have a large-scale head-to-head comparison study, the preventive effects for HBV-related HCC of first-line NAs remain unsettled.

The adherence to NA treatment also influences the protective effect of HCC prevention. In a long-term follow-up study of $\mathrm{CHB}$ patients receiving naïve ETV treatment, poor adherence significantly increased the risk of HCC development with a HR of 2.86 (95\%CI: 1.76-4.64; $P<0.001$ ), particularly among patients with cirrhosis $^{[71]}$.

\section{Non-anti-HBV therapy}

Although hepatitis B viral suppression with long-term treatment with NAs reduced the incidences of HCC, HCC risk persists in HBV carriers, especially those with advanced fibrosis. Thus, effective non-anti-HBV therapy to prevent HCC is urgently needed.

The chemoprotective effects of aspirin against cancers have been addressed for decades, including gastrointestinal tract-related cancer and lung cancer $^{[72,73]}$. Recently, the Taiwan nationwide cohort study compared 2,123 patients with daily aspirin for 90 or more days and 8,492 patients without aspirin therapy, the investigators found that aspirin therapy significantly decreased HCC risk (HR: $0.71 ; 95 \%$ CI: 0.580.86; $P<0.001)^{[74]}$. In the Swedish nationwide cohort including chronic hepatitis $\mathrm{B}$ and $\mathrm{C}$ patients, the use of aspirin decreased the cumulative incidence of HCC (aHR: 0.69; 95\%CI: 0.62-0.76). In addition, the protective effect was associated with duration of aspirin treatment ${ }^{[75]}$.

Statins, 3-hydroxy-3-methylglutaryl CoA reductase inhibitors, have potential anticancer effects, such as anti-inflammation, antiproliferation, and anti-invasion, which are associated with carcinogenesis ${ }^{[76]}$. In an earlier hospital-based study from Hong Kong, statin use reduced HCC risk by $32 \%$ in patients with chronic HBV infection ${ }^{[7]}$. In the Swedish nationwide cohort including chronic hepatitis B and C patients, the use of lipophilic statins decreased the cumulative incidence of HCC (aHR: 0.41; 95\%CI: 0.32-0.61) ${ }^{[78]}$. A recent meta-analysis, including 7 studies with HBV carriers revealed that statin therapy reduced the risk of HBVrelated HCC with a relative risk of $0.52(95 \% \mathrm{CI}: 0.40-0.68 ; P<0.001)^{[79]}$.

Although the chemopreventive effects of these non-anti-HBV drugs are encouraging, further prospective studies or randomized trials are warranted to confirm these observations.

\section{Early detection of HCC}

Early detection of HCC in HBV patients is another important strategy for secondary prevention of HCC. To identify those at high risk of HCC development, risk stratification is needed in clinical practice. Currently, several predicting models have been developed to evaluate the risk of HBV-related HCC development in CHB patients, regardless of without [Table 1] or with [Table 2] anti-HBV therapy. The components of the models included clinical features (age, sex, platelet count, serum ALT and cirrhosis) and hepatitis B viral factors (HBV DNA, HBeAg, genotype and levels of HBsAg) ${ }^{[15,16,80-94]}$. For example, there was the REACH-B model with a 17-point risk score, which included age, male sex, ALT, HBeAg-positive and HBV DNA as variables. The estimated accuracy by area under receiver operating characteristic (AUROC) curve to predict risk of HCC was 0.796 (95\%CI: 0.775-0.816) at 5 years and 0.769 (95\%CI: $0.747-0.790$ ) at 10 years ${ }^{[80]}$. The REAL-B model included $8048 \mathrm{CHB}$ patients who received oral anti-HBV treatment for prediction of the 5-year HCC risk. This model was developed on the basis of the simplified variables, including male sex, age, alcohol use, diabetes, baseline cirrhosis, platelet count, and alpha-fetoprotein (AFP). The estimated 5-year cumulative HCC incidence rates were $0.3 \%-1.38 \%$ for low risk (REAL-B score £ 3), $2.29 \%-10.24 \%$ for moderate risk (REAL-B score 4-7), and 16.5\%-90.37\% for high risk (REAL-B score 8-13). The AUROC curves for HCC risk at 3, 5, and 10 years were all greater than $0.8^{[93]}$. Based on the risk 


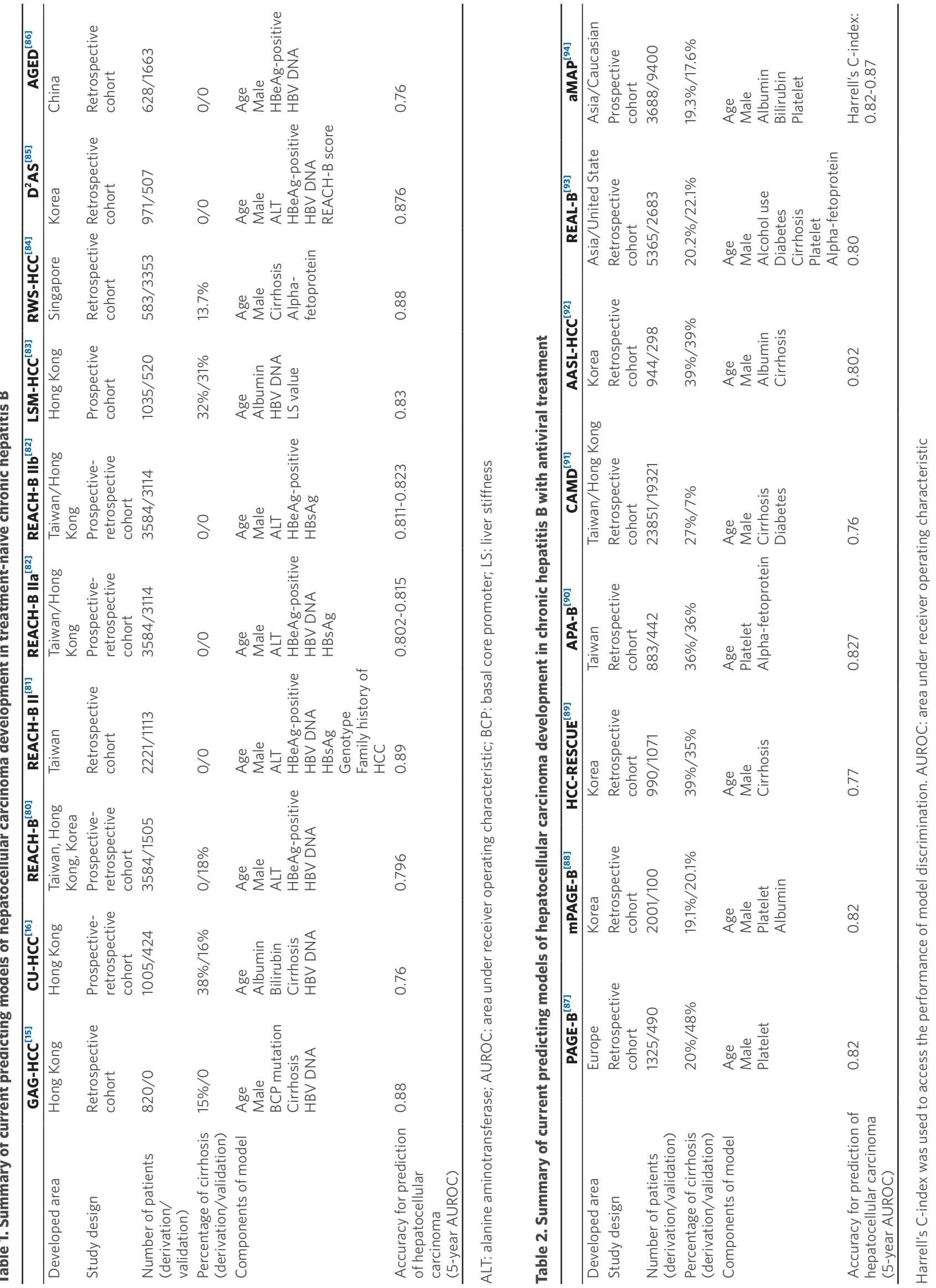


stratification through these clinical HBV-related HCC risk predicting models, HCC surveillance should be provided to HBV carriers with different risk levels. Among patients with a low HCC risk, a follow-up with regular interval is suggested. For patients with intermediate risk, a close follow-up is needed. For high-risk patients, in addition to close follow-up, anti-HBV treatment should also be considered ${ }^{[95]}$.

Currently, periodic screening by using ultrasonography with or without AFP among populations at risk of HCC is the most effective strategy for early detection of HCC in several clinical practice guidelines ${ }^{[96-99]}$. AFP is a long time and widely used biomarker for the diagnosis of HCC. However, the detection rates are only $30 \%-40 \%$ for the early- stage HCC $^{[100]}$. Protein induced by vitamin K absence II (PIVKA II) also known as Des- $\gamma$-carboxy-prothrombin was identified as a serum biomarker for HCC. In an early casecontrol study, PIVKA II had high diagnostic efficacy with a sensitivity of $61 \%$ and specificity of $70 \%$ for early stage $\mathrm{HCC}^{[101]}$. In a meta-analysis with 14 studies, the diagnostic ability of PIVKA II combined with AFP was better than that of each biomarker used alone. The sensitivity and specificity were $91 \%$ and $83 \%$, respectively. The pooled AUROC curve was 0.94 (95\%CI: $0.91-0.95)^{[102]}$. In the future, the combination of different imaging modalities and non-invasive biomarkers are likely to improve the ability of early HCC detection $^{[103]}$.

\section{Tertiary prevention}

Anti-HBV therapy

Although the advance in the treatment of HCC provides opportunities for curative treatment, a high incidence of recurrence, up to $50 \%$ at 5 years after curative treatment, was observed in patients with underlying chronic HBV infection ${ }^{[104]}$. Therefore, it is urgent to identify risk factors associated with HCC recurrence and develop tertiary prevention for HBV-related HCC after curative treatment. Ample evidence indicates that high viral load before curative treatment is associated with HCC recurrence post curative treatment ${ }^{[105,106]}$. High viral load is known to result in severe hepatic inflammatory and fibrosis scores, which are related to HCC recurrence after curative treatment ${ }^{[106]}$. Furthermore, curative treatment such as radiofrequency and surgical resection might be associated with HBV reactivation after these treatments ${ }^{[107]}$. In a retrospective study, Li et al. ${ }^{[108]}$ analyzed a cohort of $2362 \mathrm{HBV}$-related HCC patients with surgical resection and demonstrated that high preoperative viral load was associated with high incidence of microvascular invasion. Of particular note, preoperative NA treatment significantly reduced the risk of microvascular invasion and recurrence of HCC. Therefore, all CHB patients with HCC who are planning surgical treatment should receive anti-HBV therapy to prevent ongoing hepatic inflammation and HCC recurrence.

Although standard or pegylated IFN- $\alpha$ are effective in the treatment of $\mathrm{CHB}$, the effect of adjuvant IFN- $\alpha$ therapies on the prevention of HBV-related HCC recurrence after curative treatment remains unsatisfactory. In an earlier randomized controlled trial in Taiwan, the median recurrent free survival rates were similar in patients with and without adjuvant IFN- $\alpha$ therapy after curative treatment of primary HCC $^{[109]}$. Furthermore, two meta-analyses both revealed that adjuvant IFN- $\alpha$ therapy cannot decrease HCC recurrence after curative treatment ${ }^{[110,111]}$. On the contrary, NAs are more effective than IFN- $\alpha$ in preventing HCC recurrence. In a randomized controlled study, NAs treatment significantly decreased HCC recurrence after surgical resection $(\mathrm{HR}=0.48 ; 95 \% \mathrm{CI} \text { : 0.32-0.70 })^{[112]}$. Subsequently, another randomized controlled trial enrolled 200 HCC patients with or without NA treatment after surgical resection. The patients with NA treatment had better recurrence-free survival and overall survival. NA therapy significantly decreased the incidence of late recurrence $(\mathrm{HR}=0.348 \text {; 95\% CI: 0.177-0.687; } P=0.002)^{[113]}$. Several large cohort studies in Asian countries also revealed that adjuvant NA treatment significantly reduced the risk of HCC recurrence after curative treatment of primary HCC $^{[114-116]}$. Recently, a meta-analysis including 21 studies confirmed that HCC patients receiving NA therapy after curative treatment had a lower cumulative incidence of HCC recurrence with relative risk of 0.76 (95\%CI: 0.65-0.90; $P=0.001)^{[117]}$. Nowadays, NAs are recommended for the tertiary prevention of HBV-related $\mathrm{HCC}^{[98,99]}$. 


\section{Non-anti-HBV therapy}

Similar to secondary prevention, non-antiviral therapies also have a potential effect on tertiary prevention of HBV-related HCC recurrence after curative therapy. A hospital-based study, which included 430 patients with HBV-related HCC after curative therapy, revealed that aspirin reduced the risk for HCC recurrence with an aHR of $0.22-0.24^{[118]}$. A study from Korea revealed that liver transplant recipients with statin therapy had a significantly lower rate of HCC recurrence than those without statin therapy ${ }^{[119]}$. Another Japanese study with 734 HCC patients after surgical resection also demonstrated that patients with statin treatment had significantly higher recurrent-free survival than those without statin treatment ${ }^{[120]}$. The protective effect of aspirin or statin on the risk of HCC recurrence should be further validated through randomized clinical trials.

\section{THE CHALLENGES IN FIGHTING HBV-RELATED HCC}

\section{HBV is not yet curable}

Although IFN- $\alpha$ and NAs are effective in the control of disease activity in CHB, covalently closed circular DNA (cccDNA) are persistently present in HBV patients and even in those who experience HBsAg seroclearance or functional cure ${ }^{[121]}$. The challenges ahead include eliminating or at least silencing cccDNA, inhibition of virus replication and blockage of HBV intrahepatic dissemination ${ }^{[122]}$. Several novel therapeutic approaches for HBV cure have been actively investigated. The direct anti-HBV agents (DAA) include entry inhibitor targeting sodium taurocholate cotransporting polypeptide, engineered site-specific nucleases targeting cccDNA and RNA interference targeting HBV $\mathrm{RNA}^{[123]}$. The immunomodulatory host targeting agents (HTA) include innate immunity receptors agonist and adaptive immunity stimulation by therapeutic vaccine $e^{[123-125]}$. In the foreseeable future, the most promising strategies towards achieving an HBV cure will be based on a combination of DAA and $\mathrm{HTA}^{[126,127]}$.

\section{HCC risk persists even with functional cure}

It is not clear whether the risk of HCC can be completely eliminated after HBsAg seroclearance or seroconversion. In a long-term follow-up study, the estimated annual incidence of HCC was $0.31 \%$ and $0.16 \%$ in patients after spontaneous and NA-induced HBsAg seroclearance, respectively ${ }^{[128]}$. Similarly, a retrospective study from Korea revealed that the estimated annual incidence of HCC was $0.29 \%$ in noncirrhotic patients achieving HBsAg seroclearance. Notably, the estimated annual incidence of HCC was up to $2.85 \%$ in cirrhotic patients after HBsAg seroclearance ${ }^{[129]}$. These observations were also confirmed by a systemic review showing that the incidence of HCC was quite low but present in patients with HBsAg seroclearance ${ }^{[130]}$. In addition, the increased prevalence of comorbidities such as MAFLD or NAFLD, NASH and metabolic syndrome in HBV patients may be associated with HCC risk, regardless of the status of hepatitis B. In a large cohort study of Taiwanese HBV carriers, the presence of metabolic risk factors was associated with an increased risk of HCC in patients with low serum level of HBV DNA $(<2000 \mathrm{IU} / \mathrm{mL})^{[131]}$. Although effective anti-HBV therapy has led to a small proportion of patients with seroclearance or seroconversion of HBsAg, they are still at a small risk of developing HCC. Therefore, a periodic surveillance for HCC is still recommended even in patients after HBsAg seroclearance or serocoversion. This is particularly important for cirrhotic patients.

\section{CONCLUSION}

Since the discovery of HBsAg by Dr. Baruch Blumberg in the early 1960s, tremendous efforts have been made to reduce the complications, morbidity and mortality of $\mathrm{CHB}$. After three decades of global promotion, universal hepatitis B vaccination has effectively interrupted MTCT of HBV. Elimination of HBV in neonates and infants is achievable ${ }^{[35]}$. Despite the successful prevention of HBV spreading by hepatitis B vaccination, it is expected that HBV-induced cirrhosis and HCC will increase in people who have been chronically infected with HBV. Early anti-HBV treatment to prevent liver disease progression is the preferred strategy. 
Ample evidence has shown that anti-HBV therapy can reduce the risk of HCC development in patients with chronic HBV infection. However, anti-HBV therapy does not completely eliminate the risk of HCC development. Therefore, HCC surveillance is recommended in every HBV patient. Through long-term profound viral suppression by anti-HBV therapy and early detection of HCC by optimal surveillance, secondary prevention of HBV-related HCC can be reached successfully. Finally, the benefit of adjuvant anti-HBV therapy in the reduction of HCC recurrence after curative treatment makes tertiary prevention of HBV-related HCC possible. The combination of individual risk-stratification approach and three levels of preventive strategies for HBV-related HCC will substantially decrease the global burden of HBV-related HCC and improve the long-term prognosis of people living with HBV as well.

\section{DECLARATIONS}

\section{Authors' contributions}

Helped in acquisition of data, planned and conducted this project, and wrote this manuscript: Lin CL

Helped in acquisition of data, planned this project, supervised this study, obtained funding, and wrote this paper: Kao JH

\section{Availability of data and materials}

Not applicable.

\section{Financial support and sponsorship}

This work was supported by grants from the National Taiwan University Hospital, the Ministry of Health and Welfare, and the Ministry of Science and Technology, Executive Yuan, Taiwan.

\section{Conflicts of interest}

All authors declared that there are no conflicts of interest.

\section{Ethical approval and consent to participate}

Not applicable.

\section{Consent for publication}

Not applicable.

\section{Copyright}

(c) The Author(s) 2021.

\section{REFERENCES}

1. Bray F, Ferlay J, Soerjomataram I, Siegel RL, Torre LA, Jemal A. Global cancer statistics 2018: GLOBOCAN estimates of incidence and mortality worldwide for 36 cancers in 185 countries. CA Cancer J Clin 2018;68:394-424.

2. Rawla P, Sunkara T, Muralidharan P, Raj JP. Update in global trends and aetiology of hepatocellular carcinoma. Contemp Oncol (Pozn) 2018;22:141-50.

3. Goossens N, Sun X, Hoshida Y. Molecular classification of hepatocellular carcinoma: potential therapeutic implications. Hepat Oncol 2015;2:371-9.

4. Llovet JM, Montal R, Sia D, Finn RS. Molecular therapies and precision medicine for hepatocellular carcinoma. Nat Rev Clin Oncol 2018;15:599-616.

5. Yang JD, Hainaut P, Gores GJ, Amadou A, Plymoth A, Roberts LR. A global view of hepatocellular carcinoma: trends, risk, prevention and management. Nat Rev Gastroenterol Hepatol 2019;16:589-604.

6. Kao JH, Chen DS. Changing disease burden of hepatocellular carcinoma in the Far East and Southeast Asia. Liver Int 2005;25:696-703.

7. Eslam M, Sarin SK, Wong VW, et al. The Asian Pacific association for the study of the liver clinical practice guidelines for the diagnosis and management of metabolic associated fatty liver disease. Hepatol Int 2020; doi: 10.1007/s12072-020-10094-2.

8. Akinyemiju T, Abera S, Ahmed M, et al; Global Burden of Disease Liver Cancer Collaboration. The burden of primary liver cancer and underlying etiologies from 1990 to 2015 at the global, regional, and national level: results from the global burden of disease study 2015 . 
JAMA Oncol 2017;3:1683-91.

9. Levrero M, Zucman-Rossi J. Mechanisms of HBV-induced hepatocellular carcinoma. J Hepatol 2016;64:S84-101.

10. Nakagawa H, Fujita M, Fujimoto A. Genome sequencing analysis of liver cancer for precision medicine. Semin Cancer Biol 2019;55:120-7.

11. Pollicino T, Cacciola I, Saffioti F, Raimondo G. Hepatitis B virus PreS/S gene variants: pathobiology and clinical implications. J Hepatol 2014;61:408-17.

12. Fako V, Wang XW. Molecular carcinogenesis of HBV-related HCC. In: Kao JH, Chen DS, editors. Hepatitis B virus and liver disease. Singapore: Springer Nature Singapore Pte Ltd; 2018. pp. 143-62.

13. Chaturvedi VK, Singh A, Dubey SK, Hetta HF, John J, Singh MP. Molecular mechanistic insight of hepatitis B virus mediated hepatocellular carcinoma. Microb Pathog 2019;128:184-94

14. Chen CJ, Yang HI, Su J, et al; REVEAL-HBV Study Group. Risk of hepatocellular carcinoma across a biological gradient of serum hepatitis B virus DNA level. JAMA 2006;295:65-73.

15. Yuen MF, Tanaka Y, Fong DY, et al. Independent risk factors and predictive score for the development of hepatocellular carcinoma in chronic hepatitis B. J Hepatol 2009;50:80-8.

16. Wong VW, Chan SL, Mo F, et al. Clinical scoring system to predict hepatocellular carcinoma in chronic hepatitis B carriers. J Clin Oncol 2010;28:1660-5.

17. Liu CJ, Chen BF, Chen PJ, et al. Role of hepatitis B viral load and basal core promoter mutation in hepatocellular carcinoma in hepatitis B carriers. J Infect Dis 2006;193:1258-65.

18. Liu CJ, Chen BF, Chen PJ, et al. Role of hepatitis B virus precore/core promoter mutations and serum viral load on noncirrhotic hepatocellular carcinoma: a case-control study. J Infect Dis 2006;194:594-9.

19. Chen G, Lin W, Shen F, Iloeje UH, London WT, Evans AA. Past HBV viral load as predictor of mortality and morbidity from HCC and chronic liver disease in a prospective study. Am J Gastroenterol 2006;101:1797-803.

20. Yu MW, Yeh SH, Chen PJ, et al. Hepatitis B virus genotype and DNA level and hepatocellular carcinoma: a prospective study in men. $J$ Natl Cancer Inst 2005;97:265-72.

21. Lin CL, Kao JH. Hepatitis B virus genotypes and variants. Cold Spring Harb Perspect Med 2015;5:a021436.

22. Yang HI, Yeh SH, Chen PJ, et al; REVEAL-HBV Study Group. Associations between hepatitis B virus genotype and mutants and the risk of hepatocellular carcinoma. J Natl Cancer Inst 2008;100:1134-43.

23. Lin CL, Kao JH. Natural history of acute and chronic hepatitis B: the role of HBV genotypes and mutants. Best Pract Res Clin Gastroenterol 2017;31:249-55.

24. Wong GL, Chan HL, Yiu KK, et al. Meta-analysis: the association of hepatitis B virus genotypes and hepatocellular carcinoma. Aliment Pharmacol Ther 2013;37:517-26.

25. Ching LK, Gounder PP, Bulkow L, et al. Incidence of hepatocellular carcinoma according to hepatitis B virus genotype in Alaska Native people. Liver Int 2016;36:1507-15.

26. Lin C, Kao J. Clinical Implications of hepatitis B virus variants. J Formos Med Assoc 2010;109:321-5.

27. Hsieh YH, Chang YY, Su IJ, et al. Hepatitis B virus pre-S2 mutant large surface protein inhibits DNA double-strand break repair and leads to genome instability in hepatocarcinogenesis. J Pathol 2015;236:337-47.

28. Suliman I, Abdelgelil N, Kassamali F, Hassanein TI. The effects of hepatic steatosis on the natural history of HBV infection. Clin Liver Dis 2019;23:433-50.

29. Su HJ, Kao JH, Tseng TC, et al. Pathologic findings of patients with nonalcoholic fatty liver disease and the impact of concurrent hepatitis B virus infection in Taiwan. J Formos Med Assoc 2020;119:1476-82.

30. Chan AWH, Wong GLH, Chan H, et al. Concurrent fatty liver increases risk of hepatocellular carcinoma among patients with chronic hepatitis B: steatosis and cancer in hepatitis B. J Gastroenterol Hepatol 2017;32:667-76.

31. Cho H, Chang Y, Lee JH, et al. Radiologic nonalcoholic fatty liver disease increases the risk of hepatocellular carcinoma in patients with suppressed chronic hepatitis B. J Clin Gastroenterol 2020;54:633-41.

32. Abdel-Rahman O, Helbling D, Schöb O, et al. Cigarette smoking as a risk factor for the development of and mortality from hepatocellular carcinoma: an updated systematic review of 81 epidemiological studies. J Evid Based Med 2017;10:245-54.

33. Wang YH, Chuang YH, Wu CF, et al. Smoking and hepatitis B virus-related hepatocellular carcinoma risk: the mediating roles of viral load and alanine aminotransferase. Hepatology 2019;69:1412-25.

34. An P, Xu J, Yu Y, Winkler CA. Host and viral genetic variation in HBV-related hepatocellular carcinoma. Front Genet 2018;9:261.

35. Lin CL, Kao JH. Hepatitis B: immunization and impact on natural history and cancer incidence. Gastroenterol Clin North Am 2020;49:201-14.

36. World Health Organization. Guidelines for the prevention, care and treatment of persons with chronic hepatitis B infection. WHO Publishers; 2015. Available from: https:/www.who.int/hepatitis/publications/hepatitis-b-guidelines/en/. [Last accessed on 30 Sep 2020]

37. Centers for Disease Control. Global Routine Vaccination Coverage, 2010. MMWR 2011;60;1520-22.

38. Chen CL, Yang JY, Lin SF, et al. Slow decline of hepatitis B burden in general population: results from a population-based survey and longitudinal follow-up study in Taiwan. $J$ Hepatol 2015;63:354-63.

39. Chen DS, Hsu NH, Sung JL, et al. A mass vaccination program in Taiwan against hepatitis B virus infection in infants of hepatitis B surface antigen-carrier mothers. JAMA 1987;257:2597-603.

40. Ni YH, Huang LM, Chang MH, et al. Two decades of universal hepatitis B vaccination in taiwan: impact and implication for future strategies. Gastroenterology 2007;132:1287-93. 
41. Indolfi G, Easterbrook P, Dusheiko G, et al. Hepatitis B virus infection in children and adolescents. Lancet Gastroenterol Hepatol 2019;4:466-76.

42. Chang MH, You SL, Chen CJ, et al; Taiwan Hepatoma Study Group. Long-term effects of hepatitis B immunization of infants in preventing liver cancer. Gastroenterology 2016;151:472-80.e1.

43. Hung GY, Horng JL, Yen HJ, Lee CY, Lin LY. Changing incidence patterns of hepatocellular carcinoma among age groups in Taiwan. $J$ Hepatol 2015;63:1390-6.

44. Qu C, Chen T, Fan C, et al. Efficacy of neonatal HBV vaccination on liver cancer and other liver diseases over 30-year follow-up of the Qidong hepatitis B intervention study: a cluster randomized controlled trial. PLoS Med 2014;11:e1001774.

45. Yeo Y, Gwack J, Kang S, et al. Viral hepatitis and liver cancer in Korea: an epidemiological perspective. Asian Pac J Cancer Prev 2013;14:6227-31.

46. McMahon BJ, Bulkow LR, Singleton RJ, et al. Elimination of hepatocellular carcinoma and acute hepatitis B in children 25 years after a hepatitis B newborn and catch-up immunization program. Hepatology 2011;54:801-7.

47. Kao JH. Hepatitis B vaccination and prevention of hepatocellular carcinoma. Best Pract Res Clin Gastroenterol 2015;29:907-17.

48. Wen WH, Chang MH, Zhao LL, et al. Mother-to-infant transmission of hepatitis B virus infection: significance of maternal viral load and strategies for intervention. J Hepatol 2013;59:24-30.

49. Chen HL, Lee CN, Chang CH, et al; Taiwan Study Group for the Prevention of Mother-to-Infant Transmission of HBV (PreMIT Study), Taiwan Study Group for the Prevention of Mother-to-Infant Transmission of HBV PreMIT Study. Efficacy of maternal tenofovir disoproxil fumarate in interrupting mother-to-infant transmission of hepatitis B virus. Hepatology 2015;62:375-86.

50. Pan CQ, Duan Z, Dai E, et al. Tenofovir to prevent hepatitis B transmission in mothers with high viral load. $N$ Engl J Med 2016;374:2324-34.

51. Terrault NA, Lok AS, Mcmahon BJ, et al. Update on prevention, diagnosis, and treatment of chronic hepatitis B: AASLD 2018 hepatitis B guidance. Hepatology 2018;67:1560-99.

52. Lampertico P, Agarwal K, Berg T, et al. EASL 2017 Clinical Practice Guidelines on the management of hepatitis B virus infection. $J$ Hepatol 2017;67:370-98.

53. Splawn L, Bailey C, Medina J, Cho J. Heplisav-B vaccination for the prevention of hepatitis B virus infection in adults in the United States. Drugs Today 2018;54:399.

54. Sarin SK, Kumar M, Lau GK, et al. Asian-pacific clinical practice guidelines on the management of hepatitis B: a 2015 update. Hepatol Int 2016;10:1-98.

55. Suk-fong Lok A. Hepatitis B treatment: what we know now and what remains to be researched: hepatology communications. Hepatol Commun 2019;3:8-19.

56. Sung JJ, Tsoi KK, Wong VW, Li KC, Chan HL. Meta-analysis: treatment of hepatitis B infection reduces risk of hepatocellular carcinoma. Aliment Pharmacol Ther 2008;28:1067-77.

57. Miyake Y, Kobashi H, Yamamoto K. Meta-analysis: the effect of interferon on development of hepatocellular carcinoma in patients with chronic hepatitis B virus infection. J Gastroenterol 2009;44:470-5.

58. Yang YF, Zhao W, Zhong YD, Xia HM, Shen L, Zhang N. Interferon therapy in chronic hepatitis B reduces progression to cirrhosis and hepatocellular carcinoma: a meta-analysis. J Viral Hepat 2009;16:265-71.

59. Wong GL, Yiu KK, Wong VW, Tsoi KK, Chan HL. Meta-analysis: reduction in hepatic events following interferon-alfa therapy of chronic hepatitis B. Aliment Pharmacol Ther 2010;32:1059-68.

60. Lin CL, Kao JH. Review article: the prevention of hepatitis B-related hepatocellular carcinoma. Aliment Pharmacol Ther 2018;48:5-14.

61. Liu K, Choi J, Le A, et al. Tenofovir disoproxil fumarate reduces hepatocellular carcinoma, decompensation and death in chronic hepatitis B patients with cirrhosis. Aliment Pharmacol Ther 2019;50:1037-48.

62. Nguyen MH, Yang HI, Le A, et al. Reduced incidence of hepatocellular carcinoma in cirrhotic and noncirrhotic patients with chronic hepatitis B treated with tenofovir-A propensity score-matched study. J Infect Dis 2019;219:10-8.

63. Hsu YC, Ho HJ, Lee TY, et al. Temporal trend and risk determinants of hepatocellular carcinoma in chronic hepatitis B patients on entecavir or tenofovir. $J$ Viral Hepat 2018;25:543-51.

64. Choi J, Kim HJ, Lee J, Cho S, Ko MJ, Lim YS. Risk of hepatocellular carcinoma in patients treated with entecavir vs tenofovir for chronic hepatitis B: a korean nationwide cohort study. JAMA Oncol 2019;5:30-6.

65. Zhang Z, Zhou Y, Yang J, Hu K, Huang Y. The effectiveness of TDF versus ETV on incidence of HCC in CHB patients: a meta analysis. BMC Cancer 2019;19:511.

66. Wu IT, Hu TH, Hung CH, et al. Comparison of the efficacy and safety of entecavir and tenofovir in nucleos(t)ide analogue-naive chronic hepatitis B patients with high viraemia: a retrospective cohort study. Clin Microbiol Infect 2017;23:464-9.

67. Kim SU, Seo YS, Lee HA, et al. A multicenter study of entecavir vs. tenofovir on prognosis of treatment-naïve chronic hepatitis B in South Korea. J Hepatol 2019;71:456-64.

68. Lee SW, Kwon JH, Lee HL, et al. Comparison of tenofovir and entecavir on the risk of hepatocellular carcinoma and mortality in treatment-naïve patients with chronic hepatitis B in Korea: a large-scale, propensity score analysis. Gut 2020;69:1301-8.

69. Hsu YC, Wong GL, Chen CH, et al. Tenofovir versus entecavir for hepatocellular carcinoma prevention in an international consortium of chronic hepatitis B. Am J Gastroenterol 2020;115:271-80.

70. Tseng C, Hsu Y, Chen T, et al. Hepatocellular carcinoma incidence with tenofovir versus entecavir in chronic hepatitis B: a systematic review and meta-analysis. Lancet Gastroenterol Hepatol 2020;5:1039-52.

71. Shin JW, Jung SW, Lee SB, et al. Medication nonadherence increases hepatocellular carcinoma, cirrhotic complications, and mortality in 
chronic hepatitis B patients treated with Entecavir. Am J Gastroenterol 2018;113:998-1008.

72. Rothwell PM, Wilson M, Elwin C, et al. Long-term effect of aspirin on colorectal cancer incidence and mortality: 20-year follow-up of five randomised trials. Lancet 2010;376:1741-50.

73. Tsoi KKF, Ho JMW, Chan FCH, Sung JJY. Long-term use of low-dose aspirin for cancer prevention: a 10-year population cohort study in Hong Kong. Int J Cancer 2019;145:267-73.

74. Lee TY, Hsu YC, Tseng HC, et al. Association of daily aspirin therapy with risk of hepatocellular carcinoma in patients with chronic hepatitis B. JAMA Intern Med 2019;179:633-40.

75. Simon TG, Duberg AS, Aleman S, Chung RT, Chan AT, Ludvigsson JF. Association of aspirin with hepatocellular carcinoma and liverrelated mortality. N Engl J Med 2020;382:1018-28.

76. Altwairgi AK. Statins are potential anticancerous agents (review). Oncol Rep 2015;33:1019-39.

77. Hsiang JC, Wong GL, Tse YK, Wong VW, Yip TC, Chan HL. Statin and the risk of hepatocellular carcinoma and death in a hospitalbased hepatitis B-infected population: A propensity score landmark analysis. J Hepatol 2015;63:1190-7.

78. Simon TG, Duberg AS, Aleman S, et al. Lipophilic statins and risk for hepatocellular carcinoma and death in patients with chronic viral hepatitis: results from a nationwide swedish population. Ann Intern Med 2019;171:318-27.

79. Li X, Sheng L, Liu L, Hu Y, Chen Y, Lou L. Statin and the risk of hepatocellular carcinoma in patients with hepatitis B virus or hepatitis C virus infection: a meta-analysis. BMC Gastroenterol 2020;20:98.

80. Yang H, Yuen M, Chan HL, et al. Risk estimation for hepatocellular carcinoma in chronic hepatitis B (REACH-B): development and validation of a predictive score. Lancet Oncol 2011;12:568-74.

81. Lee MH, Yang HI, Liu J, et al; R.E.V.E.A.L.-HBV Study Group. Prediction models of long-term cirrhosis and hepatocellular carcinoma risk in chronic hepatitis B patients: risk scores integrating host and virus profiles. Hepatology 2013;58:546-54.

82. Yang HI, Tseng TC, Liu J, et al. Incorporating serum level of hepatitis b surface antigen or omitting level of hepatitis B virus DNA does not affect calculation of risk for hepatocellular carcinoma in patients without cirrhosis. Clin Gastroenterol Hepatol 2016;14:461-8.e2.

83. Wong GL, Chan HL, Wong CK, et al. Liver stiffness-based optimization of hepatocellular carcinoma risk score in patients with chronic hepatitis B. J Hepatol 2014;60:339-45.

84. Poh Z, Shen L, Yang HI, et al. Real-world risk score for hepatocellular carcinoma (RWS-HCC): a clinically practical risk predictor for HCC in chronic hepatitis B. Gut 2016;65:887-8.

85. Sinn DH, Lee JH, Kim K, et al. A novel model for predicting hepatocellular carcinoma development in patients with chronic hepatitis B and normal alanine aminotransferase levels. Gut Liver 2017;11:528-34.

86. Fan C, Li M, Gan Y, et al. A simple AGED score for risk classification of primary liver cancer: development and validation with longterm prospective HBsAg-positive cohorts in Qidong, China. Gut 2019;68:948-9.

87. Papatheodoridis G, Dalekos G, Sypsa V, et al. PAGE-B predicts the risk of developing hepatocellular carcinoma in Caucasians with chronic hepatitis B on 5-year antiviral therapy. J Hepatol 2016;64:800-6.

88. Kim JH, Kim YD, Lee M, et al. Modified PAGE-B score predicts the risk of hepatocellular carcinoma in Asians with chronic hepatitis B on antiviral therapy. $J$ Hepatol 2018;69:1066-73.

89. Sohn W, Cho JY, Kim JH, et al. Risk score model for the development of hepatocellular carcinoma in treatment-naïve patients receiving oral antiviral treatment for chronic hepatitis B. Clin Mol Hepatol 2017;23:170-8.

90. Chen CH, Lee CM, Lai HC, et al. Prediction model of hepatocellular carcinoma risk in Asian patients with chronic hepatitis B treated with entecavir. Oncotarget 2017;8:92431-41.

91. Hsu YC, Yip TC, Ho HJ, et al. Development of a scoring system to predict hepatocellular carcinoma in Asians on antivirals for chronic hepatitis B. J Hepatol 2018;69:278-85.

92. Yu JH, Suh YJ, Jin YJ, et al. Prediction model for hepatocellular carcinoma risk in treatment-naive chronic hepatitis B patients receiving entecavir/tenofovir. Eur J Gastroenterol Hepatol 2019;31:865-72.

93. Yang HI, Yeh ML, Wong GL, et al. Real-world effectiveness from the Asia pacific rim liver consortium for HBV risk score for the prediction of hepatocellular carcinoma in chronic hepatitis B patients treated with oral antiviral therapy. J Infect Dis2020;221:389-99.

94. Fan R, Papatheodoridis G, Sun J, et al. aMAP risk score predicts hepatocellular carcinoma development in patients with chronic hepatitis. J Hepatol 2020;73:1368-78.

95. Lin CL, Kao JH. Risk stratification for hepatitis B virus related hepatocellular carcinoma. J Gastroenterol Hepatol 2013;28:10-7.

96. Bruix J, Sherman M; American Association for the Study of Liver Diseases. Management of hepatocellular carcinoma: an update. Hepatology 2011;53:1020-2.

97. European Association For The Study Of The Liver, European Organisation For Research And Treatment Of Cancer. EASL-EORTC clinical practice guidelines: management of hepatocellular carcinoma. $J$ Hepatol 2012;56:908-43.

98. Omata M, Cheng AL, Kokudo N, et al. Asia-pacific clinical practice guidelines on the management of hepatocellular carcinoma: a 2017 update. Hepatol Int 2017;11:317-70.

99. Surveillance group, Diagnosis group, Staging group, Surgery group, Local ablation group, TACE/TARE/HAI group, Target therapy/ systemic therapy group, Radiotherapy group, Prevention group, Drafting group. Management consensus guideline for hepatocellular carcinoma: 2016 updated by the Taiwan Liver Cancer Association and the Gastroenterological Society of Taiwan. J Formos Med Assoc 2018;117:381-403.

100. Trevisani F, D'intino PE, Morselli-labate AM, et al. Serum $\alpha$-fetoprotein for diagnosis of hepatocellular carcinoma in patients with chronic liver disease: influence of HBsAg and anti-HCV status. J Hepatol 2001;34:570-5.

101. Marrero JA, Feng Z, Wang Y, et al. $\alpha$-Fetoprotein, des- $\gamma$ carboxyprothrombin, and lectin-bound $\alpha$-fetoprotein in early hepatocellular 
carcinoma. Gastroenterology 2009;137:110-8.

102. Fu J, Li Y, Li Z, Li N. Clinical utility of decarboxylation prothrombin combined with $\alpha$-fetoprotein for diagnosing primary hepatocellular carcinoma. Biosci Rep 2018;38:BSR20180044.

103. Wang W, Wei C. Advances in the early diagnosis of hepatocellular carcinoma. Genes Dis 2020;7:308-19.

104. Sasaki K, Shindoh J, Nishioka Y, et al. Impact of viral etiology on postoperative de novo recurrence after hepatectomy for hepatocellular carcinoma in cirrhotic patients. J Gastrointest Surg 2017;21:487-95.

105. Hung IFN, Poon RTP, Lai C, Fung J, Fan S, Yuen M. Recurrence of hepatitis B-related hepatocellular carcinoma is associated with high viral load at the time of resection. The American Journal of Gastroenterology 2008;103:1663-73.

106. Wu J, Huang Y, Chau G, et al. Risk factors for early and late recurrence in hepatitis B-related hepatocellular carcinoma. J Hepatol 2009;51:890-7.

107. Dan J, Zhang Y, Huang J, et al. Hepatitis B virus reactivation after radiofrequency ablation or hepatic resection for HBV-related small hepatocellular carcinoma: a retrospective study. Eur J Surg Oncol 2013;39:865-72.

108. Li Z, Lei Z, Xia Y, et al. Association of preoperative antiviral treatment with incidences of microvascular invasion and early tumor recurrence in hepatitis B virus-related hepatocellular carcinoma. JAMA Surg 2018;153:e182721.

109. Chen LT, Chen MF, Li LA, et al. Disease committee of adjuvant therapy for postoperative hepatocellular carcinoma, Taiwan Cooperative Oncology Group, National Health Research Institutes, Zhunan, Taiwan. Long-term results of a randomized, observation-controlled, phase III trial of adjuvant interferon Alfa-2b in hepatocellular carcinoma after curative resection. Ann Surg 2012;255:8-17.

110. Zhang W, Song T, Zhang T, et al. Adjuvant interferon for early or late recurrence of hepatocellular carcinoma and mortality from hepatocellular carcinoma following curative treatment: a meta-analysis with comparison of different types of hepatitis. Mol Clin Oncol 2014;2:1125-34.

111. Xu J, Li J, Chen J, Liu Z. Effect of adjuvant interferon therapy on hepatitis B/C virus-related hepatocellular carcinoma after curative therapy - meta-analysis. Adv Clin Exp Med 2015;24:331-40.

112. Yin J, Li N, Han Y, et al. Effect of antiviral treatment with nucleotide/nucleoside analogs on postoperative prognosis of hepatitis B virusrelated hepatocellular carcinoma: a two-stage longitudinal clinical study. JCO 2013;31:3647-55.

113. Huang G, Lau WY, Wang Z, et al. Antiviral therapy improves postoperative survival in patients with hepatocellular carcinoma: a randomized controlled trial. Ann Surg 2015;261:56-66.

114. Wu C, Chen Y, Ho HJ, et al. Association between nucleoside analogues and risk of hepatitis B virus-related hepatocellular carcinoma recurrence following liver resection. JAMA 2012;308:1906.

115. Lee T, Lin J, Zeng Y, Chen Y, Wu M, Wu C. Association between nucleos(t)ide analog and tumor recurrence in hepatitis B virus-related hepatocellular carcinoma after radiofrequency ablation. Hepatology 2016;63:1517-27.

116. Wong GL, Tse YK, Chan HL, Yip TC, Tsoi KK, Wong VW. Oral nucleos(t)ide analogues reduce recurrence and death in chronic hepatitis B-related hepatocellular carcinoma. Aliment Pharmacol Ther 2016;43:802-13.

117. Zhang G, Yu X, Liu P, Huang X, Jiang X. Efficacy of nucleoside analogs for chronic hepatitis B virus-related hepatocellular carcinoma after curative treatment: a meta-analysis. Dig Dis Sci 2018;63:3207-19.

118. Young SH, Chau GY, Lee IC, et al. Aspirin is associated with low recurrent risk in hepatitis B virus-related hepatocellular carcinoma patients after curative resection. J Formos Med Assoc 2020;119:218-29.

119. Cho Y, Kim MS, Nam CM, Kang ES. Statin use is associated with decreased hepatocellular carcinoma recurrence in liver transplant patients. Sci Rep 2019;9:1467.

120. Kawaguchi Y, Sakamoto Y, Ito D, et al. Statin use is associated with a reduced risk of hepatocellular carcinoma recurrence after initial liver resection. BST 2017;11:574-80.

121. Yang H, Kao J. Persistence of hepatitis B virus covalently closed circular DNA in hepatocytes: molecular mechanisms and clinical significance. Emerg Microbes Infect 2019;3:1-7.

122. Zeisel MB, Lucifora J, Mason WS, et al. Towards an HBV cure: state-of-the-art and unresolved questions-report of the ANRS workshop on HBV cure. Gut 2015;64:1314-26.

123. Lin C, Kao J. Review article: novel therapies for hepatitis B virus cure - advances and perspectives. Aliment Pharmacol Ther 2016;44:213-22.

124. Lang J, Neumann-haefelin C, Thimme R. Immunological cure of HBV infection. Hepatol Int 2019;13:113-24.

125. Smolders EJ, Burger DM, Feld JJ, Kiser JJ. Review article: clinical pharmacology of current and investigational hepatitis B virus therapies. Aliment Pharmacol Ther 2020;51:231-43.

126. Revill PA, Chisari FV, Block JM, et al. A global scientific strategy to cure hepatitis B. Lancet Gastroenterol Hepatol 2019;4:545-58.

127. Cox AL, El-sayed MH, Kao J, et al. Progress towards elimination goals for viral hepatitis. Nat Rev Gastroenterol Hepatol 2020;17:533-42.

128. Chen YC, Jeng WJ, Chien RN, Chu CM, Liaw YF. Clinical outcomes after spontaneous and nucleos(t)ide analogue-treated HBsAg seroclearance in chronic HBV infection. Aliment Pharmacol Ther 2016;43:1311-8.

129. Kim GA, Lee HC, Kim MJ, et al. Incidence of hepatocellular carcinoma after HBsAg seroclearance in chronic hepatitis B patients: a need for surveillance. J Hepatol 2015;62:1092-9.

130. Kuang XJ, Jia RR, Huo RR, et al. Systematic review of risk factors of hepatocellular carcinoma after hepatitis B surface antigen seroclearance. J Viral Hepat 2018;25:1026-37.

131. Yu M, Lin C, Liu C, Yang S, Tseng Y, Wu C. Influence of metabolic risk factors on risk of hepatocellular carcinoma and liver-related death in men with chronic hepatitis B: a large cohort study. Gastroenterology 2017;153:1006-17.e5. 